University of Wollongong

Research Online

Faculty of Engineering - Papers (Archive)

Faculty of Engineering and Information

Sciences

2005

\title{
Evaluation of Transmission Methodology for the microPET Focus 220
} Animal Scanner

W. Lehnert

University of Wollongong

S. R. Meikle

University of Sydney

S. Siegel

CTI/Concorde, Tennessee, USA

D. Bailey

CTI/Concorde, Tennessee, USA

R. Banati

University of Sydney

See next page for additional authors

Follow this and additional works at: https://ro.uow.edu.au/engpapers

Part of the Engineering Commons

https://ro.uow.edu.au/engpapers/68

\section{Recommended Citation}

Lehnert, W.; Meikle, S. R.; Siegel, S.; Bailey, D.; Banati, R.; and Rosenfeld, Anatoly B.: Evaluation of Transmission Methodology for the microPET Focus 220 Animal Scanner 2005.

https://ro.uow.edu.au/engpapers/68 


\section{Authors}

W. Lehnert, S. R. Meikle, S. Siegel, D. Bailey, R. Banati, and Anatoly B. Rosenfeld 


\title{
Evaluation of Transmission Methodology for the microPET Focus 220 Animal Scanner
}

\author{
Wencke Lehnert, Steven R. Meikle, Senior Member, IEEE, Stefan Siegel, Member, IEEE, David Bailey, Member, \\ IEEE, Richard Banati, Anatoly B. Rosenfeld, Senior Member, IEEE
}

\begin{abstract}
Attenuation Correction is important among other corrections for quantitative Positron Emission Tomography (PET). A common method is to acquire transmission data using an external source from which attenuation correction factors are derived. The aim of this work was to compare different transmission methodologies for the microPET Focus 220 animal scanner in terms of accuracy, signal-to-noise and scatter. This study included experiments in coincidence mode with and without rod windowing, singles mode with two different energy sources $\left({ }^{68} \mathrm{Ge}\right.$ and $\left.{ }^{57} \mathrm{Co}\right)$ and post-injection transmission scanning. In addition, the effectiveness of transmission segmentation was investigated. The propagation of transmission bias and noise into the emission images was also examined.

Singles transmission scanning resulted in substantially improved signal-to-noise compared with coincidence measurements. The ${ }^{57} \mathrm{Co}$ measurements provided attenuation coefficients close to the theoretical value for an energy window of $120-125 \mathrm{keV}$, while the ${ }^{68} \mathrm{Ge}$ single measurements were degraded due to scattering from the object. Transmission scatter correction improved the accuracy for a $10 \mathrm{~cm}$ phantom but over-corrected for a mouse phantom. ${ }^{57} \mathrm{Co}$ also resulted in low bias and noise in post-injection transmission scans for emission activities up to 20 MBq. Segmentation worked most reliably for transmission data acquired with ${ }^{57} \mathrm{Co}$ but the minor improvement in accuracy of attenuation coefficients and signal-to-noise did not justify its use, particularly for small subjects. The accuracy and signal-to-noise of activity concentration measurements reflected the accuracy and signal-to-noise of transmission measurements. We conclude that ${ }^{57} \mathrm{Co}$ singles transmission scanning is the most suitable method of attenuation correction on the microPET Focus 220 animal scanner.
\end{abstract}

\section{INTRODUCTION}

The microPET Focus 220 scanner is a high resolution PET system for imaging rodents and small primates. The images can be interpreted qualitatively by comparing radiotracer accumulation in target and reference regions of the body. In many experiments, however, there is either a need for absolute

Manuscript received November 9, 2005. This work was supported by the Australian Research Council and CTI-Concorde Microsystems under ARCLinkage grant LP0560912. The Ramaciotti Imaging Centre was established with an award by the Ramaciotti National Biomedical Research Initiative to the Brain and Mind Research Institute.

W. Lehnert was with the Centre for Medical Radiation Physics, University of Wollongong, NSW, Australia. She is now with the School of Medical Radiation Sciences and the Ramaciotti Imaging Centre, Brain \& Mind Research Institute, University of Sydney, Lidcombe NSW 2141, Australia (telephone: +61 29351 9519, e-mail: wlehnert@fhs.usyd.edu.au).

S. R. Meikle and R. Banati are with the School of Medical Radiation Sciences and the Ramaciotti Imaging Centre, Brain \& Mind Research Institute, University of Sydney,

S. Siegel and David Bailey are with CTI/Concorde, Knoxville TN USA.

A. B. Rosenfeld is with the Centre for Medical Radiation Physics, University of Wollongong, NSW, Australia. quantification or else there is no readily identifiable stable reference region. In these cases, attenuation correction is important among other quantitative corrections [1].

A common method to correct for attenuation in an object is to perform a transmission scan using an external source. The conventional approach is to use a rotating ${ }^{68} \mathrm{Ge}$ rod or point source, taking into account the source position to accept coincidences only for lines of response (LORs) that pass through the source at a given moment. This technique is called rod windowing or sinogram windowing as it applies a mask to the sinogram. [2], [3]

An alternative method is to record single photons instead of coincidence events [4], [5]. In this case, LORs are formed between the known source location at any time and the photons detected by opposing detectors. The advantage of this technique is an increased count rate and hence increased signal-to-noise ratio.

In general, transmission scans are performed prior to injection of the radioisotope to be imaged. However, to reduce the time the subject spends on the scanner, methods of postinjection transmission measurement were introduced [6] - [8]. Emission contamination can be estimated by acquiring a transmission scan without a transmission source present ("Mock scan") [7], or minimized by using a source that emits photons with an energy different from $511 \mathrm{keV}$.

Transmission data have Poisson noise present. If those data are used for attenuation correction the noise will propagate into the emission data. To achieve low noise levels for short acquisition times of transmission data, methods of segmentation have been introduced (e.g. [9] - [11]) which result in an essentially noiseless image and potentially more accurate estimate of attenuation.

The aims of this study were:

- To compare different transmission methods, including coincidence and singles scanning, in terms of accuracy, signal-to-noise and scatter.

- To evaluate the effect of the different transmission methods on emission measurement accuracy and noise.

- To study the feasibility of performing transmission measurements after administration of activity to the subject.

- To evaluate the potential role of segmentation of the attenuation images in reducing bias and noise propagation (based on the method developed by Bettinardi et al [11]). 


\section{MATERIALS AND METHODS}

\section{A. Transmission Experiments}

1) Transmission Measurements with Germanium $\left({ }^{68} \mathrm{Ge}\right)$

For the studies with ${ }^{68} \mathrm{Ge}$ a point source with activity ranging from 11.5-14 MBq was used. Scans were acquired in coincidence mode with and without rod windowing, and in singles mode with $1,2,4,8$ and 16 passes of the point source through the axial field of view ( 1 pass $\approx 8 \mathrm{~min}$ ). The applied energy window was $350-750 \mathrm{keV}$. The coincidence timing window in coincidence mode was set to 6 ns. For each acquisition mode a blank scan was acquired for 4 hours.

2) Transmission Measurements with Cobalt $\left({ }^{57} \mathrm{Co}\right)$

The experiments with ${ }^{57} \mathrm{Co}$ were performed with a $163 \mathrm{MBq}$ point source. Due to different scattering properties at $122 \mathrm{keV}$ compared with $511 \mathrm{keV}$ and due to the high source activity it was necessary to find an optimal energy window width. The acquisitions included energy windows of 120-125 keV, 110$135 \mathrm{keV}, 100-150 \mathrm{keV}$ and 80-180 keV. Transmission data were acquired for 1 pass and blank scans were acquired for 1 hour.

Postinjection transmission experiments were done with the mouse phantom only. The scans were acquired for 1 pass with an energy window of 120-125 keV for different emission activities of ${ }^{18} \mathrm{~F}(2-30 \mathrm{MBq})$. No mock scan was used to remove emission contamination.

3) Phantoms and Processing

Two phantoms were used for the transmission scans:

- a uniform cylinder phantom with a diameter of $10 \mathrm{~cm}$ and a length of $10 \mathrm{~cm}$.

- a mouse sized phantom (diameter $50 \mathrm{~mm}$, length $48 \mathrm{~mm}$ ), which comprises a main fillable chamber (filled with water) and two (15 mm diameter) fillable chambers (filled with polystyrene particles and water) representing the two lungs. Both phantoms were placed on the patient bed with the phantom approximately in the centre of the field of view.

Processing details can be found in Table I.

TABLE I

PROCESSING OF THE TRANSMISSION/BLANK DATA

\begin{tabular}{ll}
\hline Acquisition & Span 47, Ring Difference 23 \\
\hline Histogramming & $\begin{array}{l}\text { 2D sinograms with Single Slice Rebinning (SSRB), } \\
\text { no smoothing, randoms correction in coincidence } \\
\text { mode, dataset with scatter correction applied in } \\
\text { singles mode and coincidence mode with windowing }\end{array}$ \\
\hline Reconstruction & 2D filtered backprojection (FBP) with a ramp filter \\
\hline
\end{tabular}

For all measurements with ${ }^{57} \mathrm{Co}$, the attenuation sinogram values were scaled to $511 \mathrm{keV}$.

\section{B. Transmission Scatter Fraction}

The scatter fraction was calculated for both phantoms, using a similar method to that described in [12]. For the cylinder phantom, the attenuation and blank sinograms obtained in the transmission experiments were used. An additional acquisition was performed for the mouse phantom with the lung compartments filled completely with water.
The measured transmission count rate $\operatorname{Tr}_{\text {meas }}(\mathrm{s})$ for each projection element $\mathrm{s}$ was averaged over all projection angles and included axial planes. The theoretical transmission count rate $\operatorname{Tr}_{\text {calc }}(\mathrm{s})$ was calculated from the blank count rate $\mathrm{B}(\mathrm{s})$ and the theoretical linear attenuation coefficient $\mu_{\text {theor }}$ for both phantoms:

$$
\operatorname{Tr}_{\text {calc }}(\mathrm{s})=\mathrm{B}(\mathrm{s}) \cdot \exp \left(-\mu_{\text {theor }} \cdot \mathrm{d}(\mathrm{s})\right)
$$

where $d(s)$ is the distance through the phantom for the projection element $\mathrm{s}$. Outside the phantom $\mathrm{d}(\mathrm{s})$ is zero.

The transmission scatter fraction (SF) was derived as:

$$
\mathrm{SF}(\mathrm{s})=\frac{\operatorname{Tr}_{\text {meas }}(\mathrm{s})-\operatorname{Tr}_{\text {calc }}(\mathrm{s})}{\operatorname{Tr}_{\text {meas }}(\mathrm{s})}
$$

\section{Segmentation}

The segmentation method introduced by Bettinardi et al. [11] is an automated adaptive clustering method. The main properties of this technique are that there are no a priori assumptions made about the number of clusters and the centroid values. It is a histogram based technique, and it is adaptive with respect to the count statistics.

The segmented attenuation images were forward projected to yield attenuation sinograms. Segmentation was only performed for the images of the mouse phantom since this phantom includes compartments of differing density.

\section{Emission Experiments}

\section{1) Phantoms and Processing}

The phantoms used for the emission measurements were:

- a uniform cylinder phantom equivalent in its shape to the cold phantom used for the transmission measurements containing $20 \mathrm{MBq}$ of ${ }^{68} \mathrm{Ge}$ at the time of the measurement.

- the mouse sized phantom with the main chamber filled with $31 \mathrm{MBq}$ of ${ }^{18} \mathrm{~F}$ in water at the commencement of the emission measurement.

Processing details of the emission data are shown in Table II. Decay correction was automatically applied to all images.

TABLE II

PROCESSING OF THE EMISSION DATA

\begin{tabular}{ll}
\hline Acquisition & Span 3, Ring Difference 47 \\
\hline Histogramming & 3D sinograms, randoms and global dead time \\
& correction, no smoothing \\
\hline Reconstruction & $\begin{array}{l}\text { Fourier rebinning and 2D filtered backprojection } \\
\text { (FBP) with a ramp filter, normalization and } \\
\text { attenuation correction, no scatter correction }\end{array}$ \\
\hline
\end{tabular}

2) Emission Scans and Attenuation Correction

The emission scans for both phantoms were acquired for 1 hour with an energy window of $350-750 \mathrm{keV}$ and a coincidence timing window of 6 ns. The emission images for both phantoms were attenuation corrected with the attenuation sinograms from the transmission scans, and the forward projected attenuation sinograms from the segmented images. 


\section{RESULTS}

A. 1) Evaluation of Transmission Measurements with ${ }^{68} \mathrm{Ge}$

In Fig. 1 and Fig. 2 the results in terms of attenuation coefficients are presented for the measurements with both phantoms and the ${ }^{68} \mathrm{Ge}$ point source.

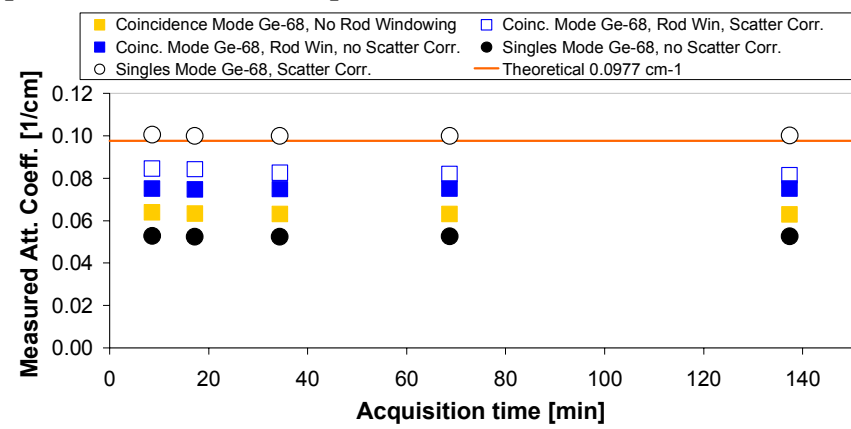

Fig. 1. Cylinder Phantom: Measured linear attenuation coefficients as a function of acquisition time for the different acquisition modes with ${ }^{68} \mathrm{Ge}$.

In the cylinder phantom the linear attenuation coefficient closest to the theoretical value was measured for singles mode with scatter correction.

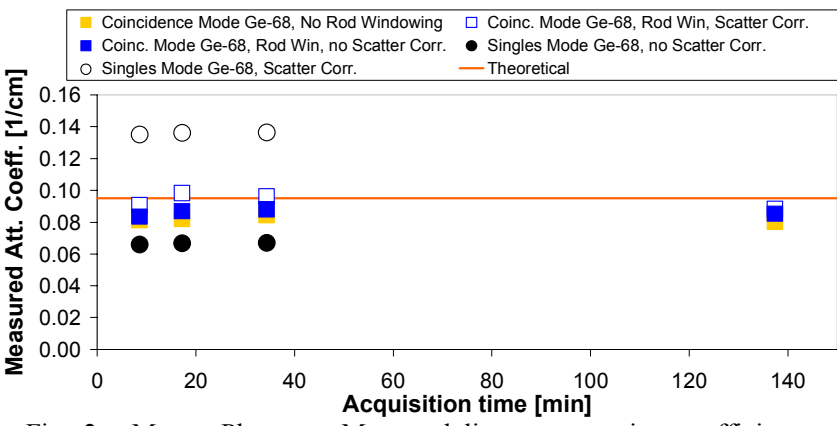

Fig. 2. Mouse Phantom: Measured linear attenuation coefficients as a function of acquisition time for the different acquisition modes with ${ }^{68} \mathrm{Ge}$.

The smallest deviation from the theoretical attenuation coefficient was achieved with coincidence mode with rod windowing and scatter correction. However, scatter correction in singles mode overcorrected the attenuation coefficients.

Fig. 3 represents the noise measured in the cylinder phantom. Since these values are comparable to the noise measured in the mouse phantom, no additional graph is displayed for the mouse phantom.

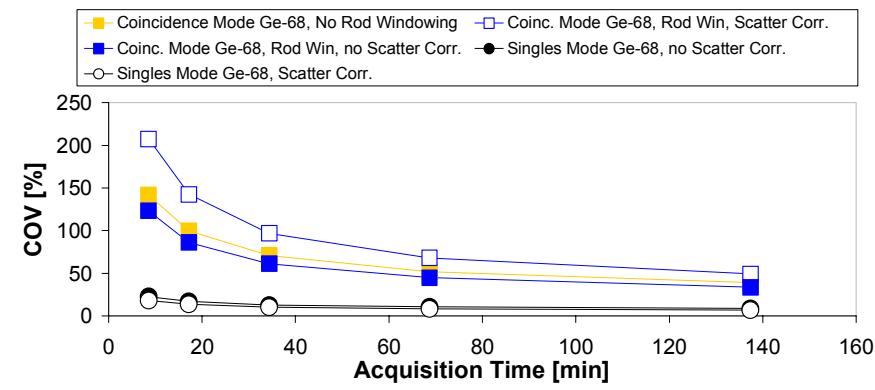

Fig. 3. Noise in the attenuation images of the cylinder phantom displayed as coefficient of variation (COV) as a function of acquisition time for the different acquisition modes with ${ }^{68} \mathrm{Ge}$.

The noise was lowest in the images acquired in singles mode (COV <23\%). High noise levels were found in coincidence mode, significantly increasing for shorter acquisition times (COV up to $207 \%$ for 1 pass).

\section{A. 2) Evaluation of Transmission Measurements with ${ }^{57} \mathrm{Co}$}

The results for the measurements with both phantoms and the ${ }^{57}$ Co point source are presented in Fig. 4.

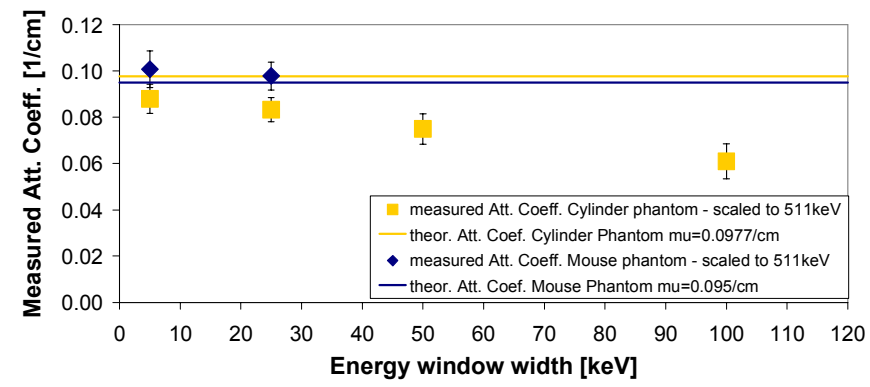

Fig. 4. Measured and scaled linear attenuation coefficients as a function of energy window width for the scans with ${ }^{57} \mathrm{Co}$. The noise in the attenuation images $(\mathrm{COV})$ is represented as error bars (1 Std. Dev.).

For the smallest energy window of 120-125 keV, the linear attenuation coefficients were reasonably close to the theoretical value after scaling to $511 \mathrm{keV}$ (bias of $10 \%$ for the cylinder phantom, $6 \%$ for the mouse phantom). But they dropped for larger energy windows. The noise level was very low for all measurements with ${ }^{57} \mathrm{Co}(\max .10 \%)$.

The graph in Fig. 5 shows the results for postinjection transmission measurements.

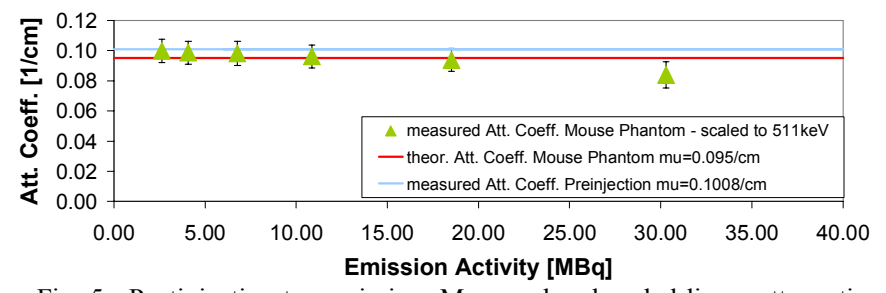

Fig. 5. Postinjection transmission: Measured and scaled linear attenuation coefficients as a function of emission activity in comparison with theoretical and measured pre-injection values.

Without application of a mock scan the deviation of the attenuation coefficients from the theoretical value is negligible for emission activities up to $20 \mathrm{MBq}$.

\section{B. Determination of the Transmission Scatter Fraction}

The results for the cylinder phantom are displayed in Fig. 6, the results for the mouse phantom in Fig. 7.

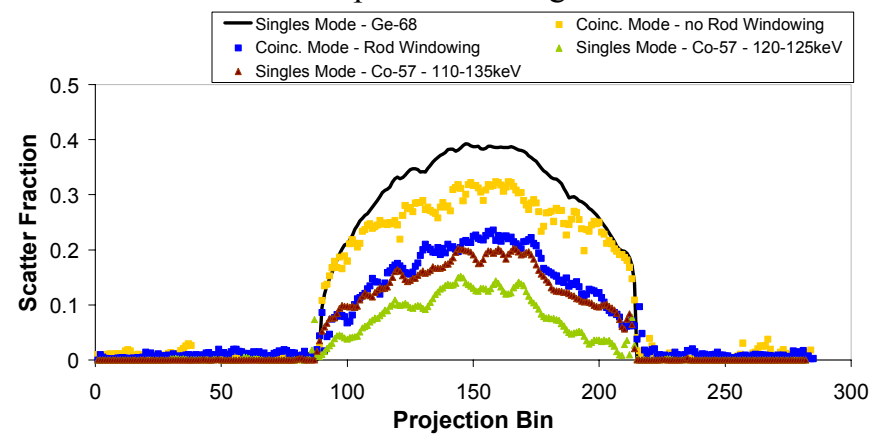

Fig. 6. Transmission scatter fraction profile for the cylinder phantom. 


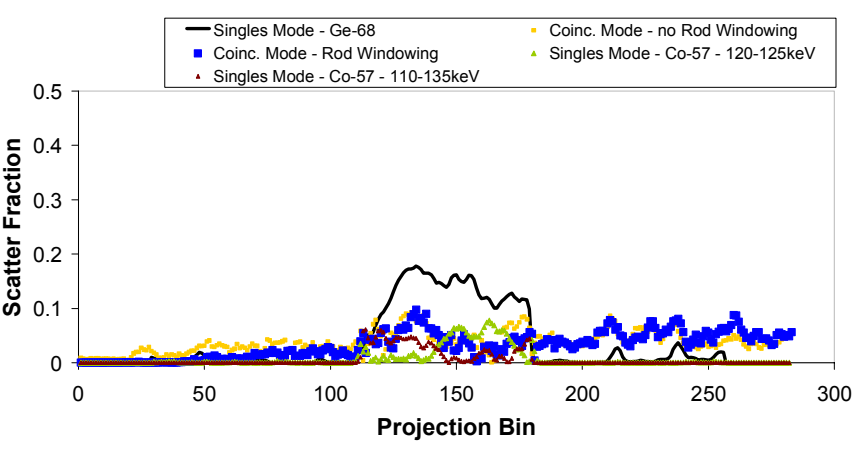

Fig. 7. Transmission scatter fraction profile for the mouse phantom.

The highest scatter in the cylinder phantom arose in singles mode with ${ }^{68} \mathrm{Ge}$ (max. $\approx 40 \%$ ), followed by coincidence mode without rod windowing (max. $\approx 32 \%$ ) and coincidence mode with rod windowing ( $\max . \approx 23 \%$ ). The lowest scatter fractions were measured with ${ }^{57} \mathrm{Co}$ for the energy windows of 120-125 $\mathrm{keV}$ (max. $\approx 15 \%$ ), and $110-135 \mathrm{keV}$ (max. $\approx 20 \%$ ).

The scatter for the mouse phantom was highest with ${ }^{68} \mathrm{Ge}$ in singles mode, as it was for the cylinder phantom, with a maximum of approx. $18 \%$. The maximum scatter fractions for coincidence mode in the mouse phantom were $9 \%$ (no rod windowing) and $10 \%$ (rod windowing). The scatter fractions were lowest for singles mode with ${ }^{57} \mathrm{Co}$.

\section{Segmentation}

Segmented images for the different transmission images of the mouse phantom are presented in Fig. 8.

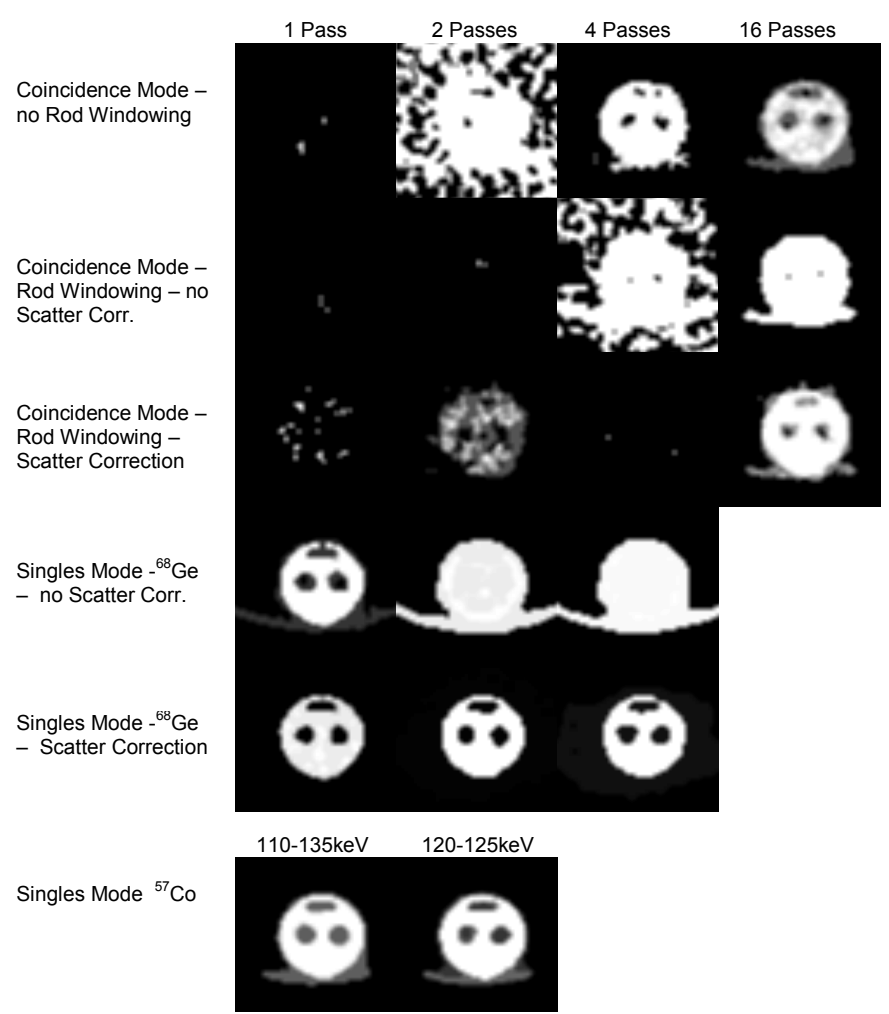

Fig. 8. Segmented images for the different transmission acquisition modes
The segmentation algorithm worked well and assigned approximately the theoretical attenuation coefficient of 0.095 $\mathrm{cm}^{-1}$ for all images produced from singles transmission images acquired with ${ }^{57} \mathrm{Co}$, and for the images acquired with ${ }^{68} \mathrm{Ge}$ in singles mode with scatter correction for acquisition times of 2 and 4 passes.

In a lot of images measured in coincidence mode, the algorithm could not identify the object or differentiate between tissue and lung. The segmented attenuation coefficients were correct in some cases and deviated by up to $42 \%$ from the theoretical value in others.

\section{Evaluation of Emission Images}

The results for the cylinder phantom are shown in Fig. 9 and Fig. 10

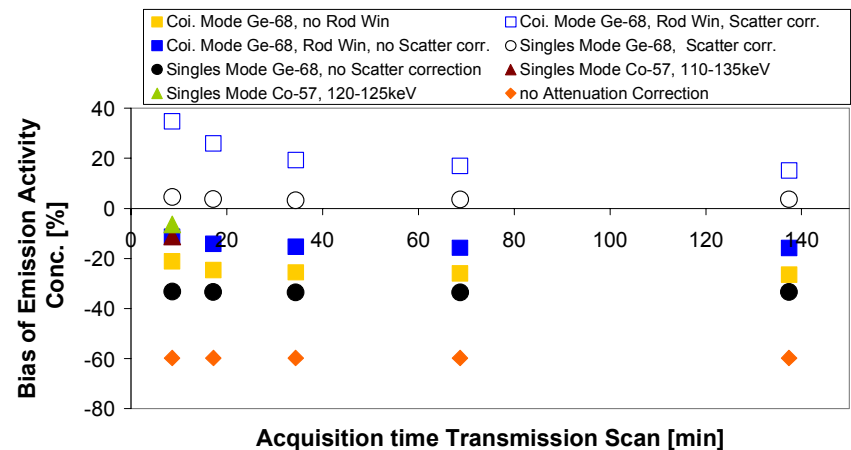

Fig. 9. Bias of emission activity concentration as a function of transmission acquisition time for the cylinder phantom.

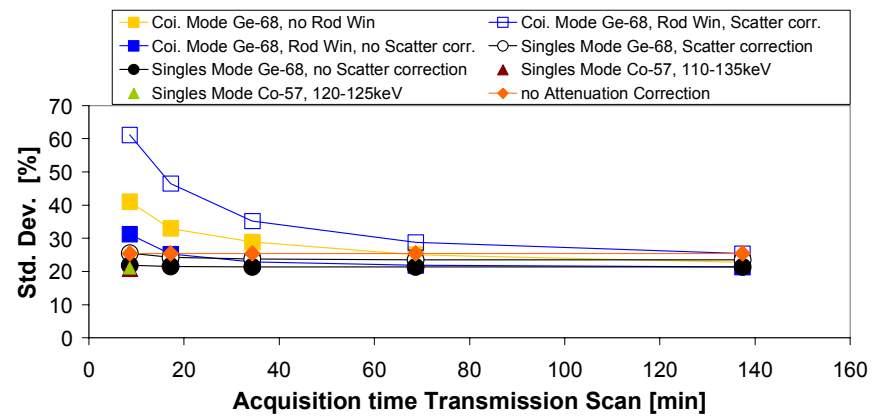

Fig. 10. Noise in the attenuation corrected emission images of the cylinder phantom as a function of transmission acquisition time.

Attenuation correction of the emission data performed with scatter corrected ${ }^{68} \mathrm{Ge}$ singles transmission data and with ${ }^{57} \mathrm{Co}$ singles transmission data (energy window 120-125 keV) resulted in a low bias $(<10 \%)$ in quantification of activity concentration. In addition, the introduced noise was low for singles transmission data acquired with ${ }^{68} \mathrm{Ge}$ and ${ }^{57} \mathrm{Co}$ compared with coincidence mode transmission data, especially for short transmission acquisition times.

The results for the mouse phantom are shown in Fig. 11 and Fig. 12. 


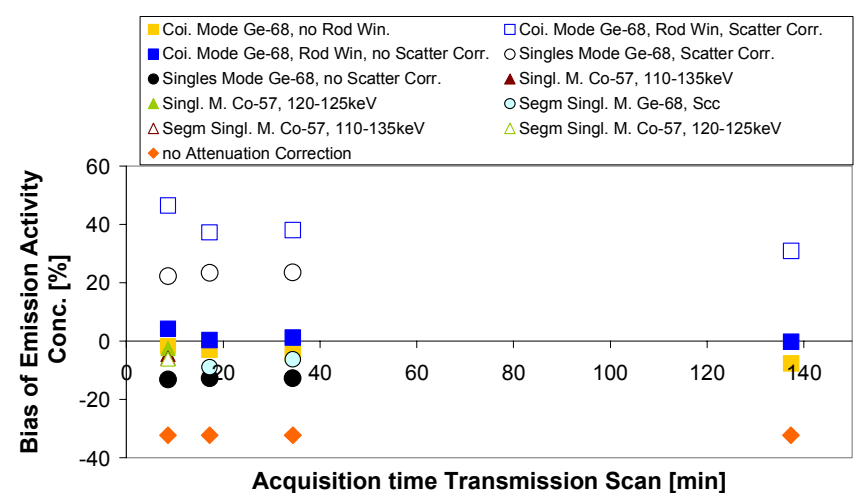

Fig. 11. Bias of emission activity concentration as a function of transmission acquisition time for the mouse phantom.

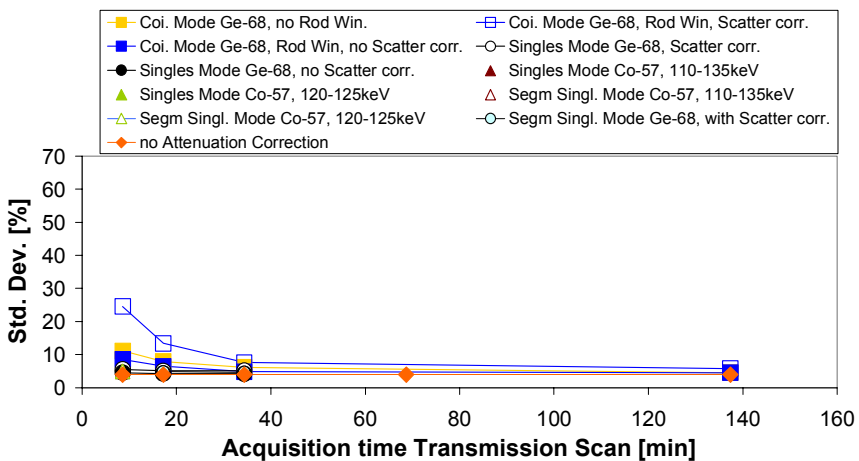

Fig. 12. Noise in the attenuation corrected emission images of the mouse phantom as a function of transmission acquisition time.

A low bias $(<10 \%)$ in emission activity concentration was achieved with attenuation correction using coincidence mode without rod windowing and with rod windowing but no scatter correction, ${ }^{57} \mathrm{Co}$ singles transmission data, and segmented transmission data. The noise levels introduced by attenuation correction with singles transmission data using ${ }^{68} \mathrm{Ge}$ and ${ }^{57} \mathrm{Co}$, and with the segmented data were low (max. $1.5 \%$ higher than for the non attenuation corrected image).

\section{CONCLUSIONS}

Singles transmission scanning results in substantially improved signal-to-noise compared with coincidence measurements. The accuracy of measured attenuation coefficients is dependent on the amount of scattering in the examined object which causes a decrease in the measured attenuation coefficients. The attenuation coefficients measured for ${ }^{57} \mathrm{Co}$ were reasonably accurate when a narrow energy window (120-125 keV) was applied, while the measurements with ${ }^{68} \mathrm{Ge}$ in singles mode had relatively high acceptance of scatter, resulting in very low attenuation coefficients $\left(0.05 \mathrm{~cm}^{-}\right.$ ${ }^{1}$ to $0.07 \mathrm{~cm}^{-1}$ ). Thus, to achieve accurate attenuation values for ${ }^{68} \mathrm{Ge}$ requires scatter correction, and the algorithm used in this study was not sufficiently accurate.

Thus, of the transmission methods studied, ${ }^{57} \mathrm{Co}$ singles measurements with a narrow energy window provide the best results in terms of noise and accuracy. This method also makes it possible to acquire transmission data post-injection of the activity to the subject. Emission contamination from $511 \mathrm{keV}$ into the energy window of $120-125 \mathrm{keV}$ was found to be negligible for activities up to $20 \mathrm{MBq}$, since no significant increase in bias or noise was observed.

Segmentation worked best for transmission data acquired with ${ }^{57} \mathrm{Co}$, delivering accurate attenuation coefficients with no noise. Problems with segmentation occurred for more noisy images, especially in coincidence mode resulting in poor differentiation between different tissues. However, segmentation is less useful for data acquired with ${ }^{57} \mathrm{Co}$, particularly for small subjects like mice since the attenuation coefficients were already close to the theoretical value and noise was acceptably low. There may be a role for segmentation in larger subjects, such as primates, where scatter causes more bias.

Our results from the emission measurements confirmed that the accuracy and signal-to-noise of activity concentration estimates is directly related to the accuracy and signal-to-noise of attenuation coefficients derived from the transmission images.

We conclude that the method of choice for attenuation correction on the microPET Focus 220 animal scanner is singles transmission scanning using a ${ }^{57} \mathrm{Co}$ point source. The potential for including segmentation of transmission data for larger subjects requires further investigation.

\section{REFERENCES}

[1] P. L. Chow et al.: "Transmission Imaging and Attenuation Correction for the microPET ${ }^{\circledast 4}$ Pomograph", IEEE Nuclear Science Symposium, vol. 2: pp. 1298-1302, 2002.

[2] R. H. Huesman et al.: "Orbiting Transmission Source for Positron Tomography", IEEE Trans. Nucl. Sci., vol. 35, no. 1: pp. 735-739, 1988.

[3] N. T. Ranger, C. J. Thompson, A. C. Evans: "The application of a masked orbiting transmission source for attenuation correction in PET", J. Nucl. Med., vol. 30: pp. 1056-1068, 1989.

[4] R. A. de Kemp, C. Nahmias: "Attenuation Correction in PET using single photon transmission measurement". Med. Phys., vol. 21, no. 6: pp. 771-778, 1994.

[5] J. S. Karp, G. Muehllehner, H. Qu, X.-H. Yan: "Singles transmission in volume-imaging PET with a ${ }^{137}$ Cs source". Phys. Med. Biol., vol. 40: pp. 929-944, 1995.

[6] R. E. Carson, M. E. Witherspoon, M. V. Green: "A Method for Postinjection PET Transmission Measurements with a Rotating Source", J. Nucl. Med., vol. 29: pp. 1558-1567, 1988.

[7] R. J. Smith, J. S. Karp: "Post Injection Transmission Scanning in a Volume Imaging PET Camera", IEEE Trans. Nucl. Sci., vol. 41, no. 4: pp. 1526-1531, 1994.

[8] P. K. Hooper, S. R. Meikle, S. Eberl, M. J. Fulham: "Validation of postinjection transmission measurements for attenuation correction in neurologic FDG PET studies", J Nucl. Med., vol. 37: pp. 128-136, 1996.

[9] E. Z. Xu, N. A. Mullani, K. L. Gould, W. L. Anderson .: "A Segmented Attenuation Correction for PET", J. Nucl. Med, vol. 32: pp.161-165, 1991.

[10] S. R. Meikle, M. Dahlbohm, S. R. Cherry .: "Attenuation Correction Using Count-Limited Transmission Data in Positron Emission Tomography”, J. Nucl. Med, vol.34: pp.143-150, 1993.

[11] V. Bettinardi et al.: "An automatic classification technique for attenuation correction in positron emission tomography", Eur. J. Nucl. Med., vol. 26: pp.447-458, 1999.

[12] D. L. Bailey, L. Livieratos, W. F. Jones, T. Jones: "Strategies for Accurate Attenuation Correction with Single Photon Transmission Measurements in 3D PET". IEEE Nuclear Science Symposium, vol. 2: pp 1009-1013, 1997. 